\title{
DETERMINAÇÃO VOLTAMÉTRICA DE CICLAMATO DE SÓDIO EM PRODUTOS DIETÉTICOS EMPREGANDO UM ELETRODO DE DIAMANTE DOPADO COM BORO
}

Roberta Antigo Medeiros, Adriana Evaristo de Carvalho, Romeu C. Rocha-Filho e Orlando Fatibello-Filho*

Departamento de Química, Universidade Federal de São Carlos, CP 676, 13560-970 São Carlos - SP, Brasil

Recebido em 30/7/07; aceito em 18/1/08; publicado na web em 13/8/08

\begin{abstract}
VOLTAMMETRIC DETERMINATION OF SODIUM CYCLAMATE IN DIETARY PRODUCTS USING A BORON-DOPED DIAMOND ELECTRODE. The use of square-wave voltammetry in conjunction with a cathodically pre-treated diamond electrode for the analytical determination of sodium cyclamate is described. The samples were analyzed as received in a $0.5 \mathrm{~mol} \mathrm{~L}^{-1} \mathrm{H}_{2} \mathrm{SO}_{4}$ solution in the concentration range from $5.0 \times 10^{-5} \mathrm{~mol} \mathrm{~L}^{-1}$ to $4.1 \times 10^{-4} \mathrm{~mol} \mathrm{~L}^{-1}$, with a detection limit of $4.8 \times 10^{-6} \mathrm{~mol} \mathrm{~L}^{-1}$. The RSD was smaller than $1.2 \%$ and the proposed method was applied with success in the determination of sodium cyclamate in several dietary products.
\end{abstract}

Keywords: sodium cyclamate; square-wave voltammetry; boron-doped diamond electrode.

\section{INTRODUÇÃO}

O ciclamato de sódio é um adoçante artificial descoberto em 1937 por Michael Sveda, um estudante de Química da Universidade de Illinois (EUA), que casualmente descobriu seu sabor adocicado. ${ }^{1}$ É um pó branco, cristalino, inodoro, 30 vezes mais doce que a sacarose, não calórico (zero caloria) e mais estável que outros adoçantes artificiais como o aspartame e a sacarina, o que possibilita sua utilização em altas e baixas temperaturas. ${ }^{2-4} \mathrm{O}$ ciclamato de sódio é comumente empregado junto com a sacarina, uma vez que o ciclamato pode mascarar o sabor residual amargo deixado pela sacarina. A utilização dessa mistura aumentou o consumo dos adoçantes artificiais nos Estados Unidos na década de 60, quando foram produzidas mais de 7,7 mil t de ciclamato. ${ }^{4}$ Porém, no fim da década de 60 , através de experimentos com ratos constatou-se que o ciclamato de sódio não era eliminado de forma invariável, mas poderia ser metabolizado como cicloexilamina. Estes experimentos ainda indicaram o aparecimento de calcificação do miocárdio, câncer de bexiga, ruptura de cromossomos e deformação de embriões, devido à presença da cicloexilamina. A partir desses estudos, o uso do ciclamato de sódio foi proibido nos EUA. ${ }^{2,4}$ Atualmente, o ciclamato de sódio é aprovado como adoçante de baixa caloria em mais de 40 países, inclusive no Brasil, Alemanha, África do Sul e Suíça. ${ }^{3}$ Os resultados de todos os estudos realizados visando verificar a toxicidade do ciclamato de sódio ou da mistura ciclamato/sacarina apresentaram poucos efeitos fisiopatológicos mesmo quando doses elevadas do edulcorante foram ingeridas. Após avaliação dos estudos toxicológicos disponíveis, considera-se seguro o uso do ciclamato em alimentos e bebidas, tendo lhe conferido a ingestão diária máxima aceitável de $11,0 \mathrm{mg} / \mathrm{kg}$ do peso corporal do consumidor. ${ }^{5}$

Sendo assim, devido a sua ampla utilização e pelas controvérsias com relação ao seu processo metabólico e toxicidade, há ainda interesse no desenvolvimento de métodos analíticos para a determinação desses edulcorantes em produtos dietéticos. Vários métodos, por exemplo, cromatográficos ${ }^{5-9}$ e espectrofotométricos, ${ }^{10-12}$ têm sido propostos para a determinação do ciclamato de sódio. Dentre esses métodos, a cromatografia líquida de alta eficiência (CLAE) é a técnica mais utilizada para a determinação deste adoçante, devido a sua

\footnotetext{
*e-mail: bello@dq.ufscar.br.
}

seletividade. Porém, esta é uma técnica de alto custo, requer maior tempo de análise e pode ser trabalhosa para algumas amostras. Isto tem despertado o interesse para as técnicas eletroanalíticas, por serem simples e de baixo custo. Assim, alguns métodos potenciométricos e amperométricos foram desenvolvidos para a determinação do ciclamato de sódio em produtos alimentícios. Richardson e Luton ${ }^{13}$ determinaram ciclamato em suco de frutas e em xaropes expectorantes, em meio fortemente ácido utilizando nitrito de sódio como titulante, sendo o ponto final destas titulações detectado amperometricamente. Já Fatibello-Filho et al. ${ }^{14}$ propuseram um procedimento simples, preciso e exato para determinação de ciclamato em produtos dietéticos como pudim, gelatina, adoçantes de mesa, coca-cola e guaraná. Neste procedimento, o ciclamato foi titulado com uma solução de nitrito de sódio em solução de ácido fosfórico $1,0 \mathrm{~mol} \mathrm{~L}^{-1} \mathrm{e}$ o ponto final da titulação foi determinado biamperometricamente. Por outro lado, um minissensor baseado na imobilização de membranas lipídicas (s-BLMs) sobre um eletrodo de prata foi desenvolvido por Nikolelis e Pantoulias ${ }^{15}$ para a determinação de acesulfame-K, ciclamato de sódio e sacarina. A correspondente curva analítica para o ciclamato de sódio foi linear num intervalo de concentração de 10 a $160 \mu \mathrm{mol}$ $\mathrm{L}^{-1}$, com limite de detecção de $10 \mu \mathrm{mol} \mathrm{L}{ }^{-1}$.

A voltametria de onda quadrada (SWV) é, entre as técnicas voltamétricas de pulso, uma das mais rápidas e sensíveis, sendo que os limites de detecção alcançados podem ser comparados aos das técnicas cromatográficas e espectrofotométricas. A análise dos resultados também possibilita a avaliação cinética e mecanística, considerandose as espécies adsorvidas ou não à superfície do eletrodo de trabalho. Além disso, a SWV tem se mostrado extremamente sensível para a detecção de moléculas orgânicas. ${ }^{16}$

Eletrodos de diamante dopado com boro (DDB) ${ }^{17}$ têm se destacado nos últimos anos por apresentarem um número importante de propriedades eletroquímicas distinguíveis das de outros eletrodos comumente usados, tais como carbono vítreo, grafite pirolítico e pasta de carbono. ${ }^{17-20}$ Propriedades como baixa e estável corrente de fundo, extraordinária estabilidade morfológica e micro-estrutural a altas temperaturas (por exemplo, $180{ }^{\circ} \mathrm{C}$ ), boa resposta a alguns analitos em soluções aquosas e não-aquosas, sem qualquer pré-tratamento convencional, fraca adsorção de moléculas polares, o que melhora a resistência do eletrodo à desativação ou envenenamento, estabilidade de resposta em longo prazo e, finalmente, uma ampla janela de 
potencial em meio aquoso e não-aquoso. ${ }^{21}$ Neste eletrodo, a janela de potencial é cerca de 3,0 V em meio aquoso, abrindo possibilidades analíticas em um grande intervalo de potenciais, especialmente positivos, permitindo estudos de oxidação não possíveis em outros eletrodos como, por exemplo, o eletrodo de mercúrio. ${ }^{21-25}$

Suffredini et al. ${ }^{19}$ chamaram a atenção para o efeito de prétratamentos eletroquímicos sobre a resposta eletroquímica do eletrodo de diamante dopado com boro. Especificamente, neste trabalho, os autores relatam que, após a realização de uma pré-polarização catódica do eletrodo de DDB, obteve-se uma melhora significativa da sua resposta eletroquímica para clorofenóis e, conseqüentemente, do limite de detecção para estes compostos.

Sendo assim, neste trabalho procurou-se combinar as vantagens do emprego da técnica SWV e aquelas do eletrodo de diamante dopado com boro pré-tratado catodicamente para o desenvolvimento de um procedimento para a determinação de ciclamato de sódio em produtos dietéticos.

\section{PARTE EXPERIMENTAL}

\section{Reagentes e soluções}

Todas as soluções foram preparadas com reagentes de grau analítico e água desionizada. Soluções padrão de ciclamato de sódio (Sigma) $1,0 \times 10^{-2} \mathrm{~mol} \mathrm{~L}^{-1}$ foram preparadas diariamente. O eletrólito suporte utilizado foi o ácido sulfúrico (Synth) $0,5 \mathrm{~mol} \mathrm{~L}^{-1}$. Previamente à realização dos experimentos, todas as soluções foram desoxigenadas por meio de borbulhamento de nitrogênio gasoso por $10 \mathrm{~min}$.

As amostras de adoçantes dietéticos líquidos $\left(\right.$ Zero cal $^{\circledR} \mathrm{e}$ Finn $\left.{ }^{\circledR}\right)$, preparado sólido para refresco diet $\left(\right.$ Magro $\left.^{\circledR}\right)$ e refrigerantes de guaraná diet (Antarctica ${ }^{\circledR} \mathrm{e} \mathrm{Kuat}^{\circledR}$ ) utilizadas para a determinação do ciclamato de sódio foram obtidas em supermercados locais.

\section{Preparo das amostras}

\section{Adoçantes dietéticos líquidos}

Alíquotas de $10 \mu \mathrm{L}$ dos adoçantes dietéticos líquidos (Zero cal ${ }^{\circledR}$ e Finn ${ }^{\circledR}$ ) foram transferidas diretamente para a célula eletroquímica contendo $10 \mathrm{~mL}$ do eletrólito suporte $\left(\mathrm{H}_{2} \mathrm{SO}_{4} 0,5 \mathrm{~mol} \mathrm{~L}^{-1}\right)$. Em seguida, os voltamogramas de onda quadrada foram obtidos.

\section{Preparado sólido para refresco}

Uma massa de 0,0670 g $\left(\right.$ Magro $\left.^{\circledR}\right)$ foi transferida para balões volumétricos de $10 \mathrm{~mL}$ e dissolvida com $\mathrm{H}_{2} \mathrm{SO}_{4} 0,5 \mathrm{~mol} \mathrm{~L}^{-1}$. Desta solução foi retirada uma alíquota de $400 \mu \mathrm{L}$, transferida diretamente para a célula eletroquímica contendo $10 \mathrm{~mL}$ do eletrólito suporte $\left(\mathrm{H}_{2} \mathrm{SO}_{4} 0,5 \mathrm{~mol} \mathrm{~L}^{-1}\right)$ e, então, os voltamogramas de onda quadrada foram obtidos.

\section{Refrigerantes de guaraná}

As duas amostras de refrigerante diet de guaraná (Antarctica ${ }^{\circledR}$ e Kuat ${ }^{\circledR}$ ) foram desgaseificadas por 5 min em banho de ultra-som. Em seguida, para obtenção dos respectivos voltamogramas de onda quadrada, volumes de $1 \mathrm{~mL}$ (Antarctica ${ }^{\circledR}$ ) e $2 \mathrm{~mL}$ (Kuat ${ }^{\circledR}$ ) foram transferidos separadamente para a célula eletroquímica contendo 10 $\mathrm{mL}$ de $\mathrm{H}_{2} \mathrm{SO}_{4} 0,5 \mathrm{~mol} \mathrm{~L}^{-1}$.

\section{Construção da curva analítica}

Após a otimização dos parâmetros experimentais do procedimento proposto, a curva analítica foi paulatinamente construída pela obtenção de voltamogramas de onda quadrada após cada adição de uma alíquota de solução padrão de ciclamato de sódio $0,01 \mathrm{~mol} \mathrm{~L}^{-1}$ à célula eletroquímica contendo $10 \mathrm{~mL}$ de solução de $\mathrm{H}_{2} \mathrm{SO}_{4} 0,5$ mol L-1.

As amostras foram analisadas empregando-se o método da adição de padrão sem nenhum tratamento prévio ou separação. As medidas foram feitas em triplicata para cada amostra.

\section{Instrumentação}

As medidas voltamétricas foram realizadas em uma célula de vidro, com capacidade de $15 \mathrm{~mL}$, equipada com uma tampa de Teflon ${ }^{\circledR}$ contendo orifícios para a desoxigenação da amostra com nitrogênio gasoso e posicionamento dos eletrodos de trabalho, de referência e auxiliar. O eletrodo de referência utilizado foi o de $\mathrm{Ag} / \mathrm{AgCl}(\mathrm{KCl}$ $3,0 \mathrm{~mol} \mathrm{~L}^{-1}$ ) e todos os potenciais relatados neste trabalho estão a ele referidos. Uma placa de platina foi usada como eletrodo auxiliar.

$\mathrm{O}$ eletrodo de trabalho utilizado nas medidas voltamétricas foi um eletrodo de diamante dopado com boro $(\mathrm{B} / \mathrm{C}=8000 \mathrm{ppm})$, com uma área de $0,72 \mathrm{~cm}^{2}$, fabricado pelo Centre Suisse de Électronique et de Microtechnique SA (CSEM), em Neuchatêl, Suíça. Previamente a seu uso, este eletrodo foi pré-tratado catodicamente para se obter uma melhor resposta com relação ao ciclamato de sódio. Optou-se por realizar este pré-tratamento galvanostaticamente, pois assim se controla exatamente a carga que circula em cada pré-tratamento, ${ }^{26}$ neste caso era aplicada uma corrente de $-0,72 \mathrm{~A}$ por $60 \mathrm{~s}$ em uma solução de $\mathrm{H}_{2} \mathrm{SO}_{4} 0,5 \mathrm{~mol} \mathrm{~L}^{-1}$.

Para a realização das medidas voltamétricas, utilizou-se um potenciostato/galvanostato Autolab PGSTAT-30 (Ecochemie) gerenciado pelo programa computacional GPES 4.0.

O método proposto foi validado usando-se a técnica de cromatografia líquida de alta eficiência (CLAE). Foi utilizado um cromatógrafo LC-10AT Shimadzu com detector de UV-VIS, modelo SPDM10AVP e coluna Shim-Pack CLC-ODS $(60 \mu \mathrm{m}$ x $150 \mathrm{~mm}, 5 \mu \mathrm{m})$. A fase móvel utilizada foi acetonitrila/tampão fosfato $(\mathrm{pH}=4)$, na proporção $14 / 86 \mathrm{v} / \mathrm{v}$, com vazão de $0,8 \mathrm{~mL}$ por min, em um volume de injeção de $30 \mu \mathrm{L}$, sendo que as espécies foram monitoradas no comprimento de onda de $200 \mathrm{~nm}$.

\section{RESULTADOS E DISCUSSÃO}

Assim como observado inicialmente por voltametria cíclica, o ciclamato de sódio apresentou somente um pico de oxidação, em torno de 1,9 V. Na Figura 1 está apresentado um voltamograma de onda quadrada para o ciclamato de sódio com as componentes de corrente. Observa-se um comportamento típico de uma reação irreversível. Podese notar que não há participação da corrente reversa e que as correntes direta e resultante são praticamente as mesmas.

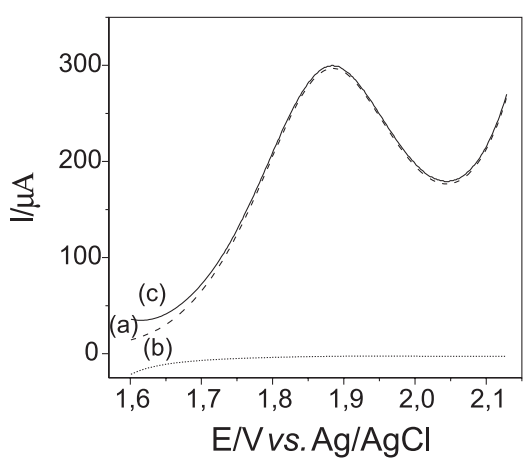

Figura 1. Voltamogramas de onda quadrada para ciclamato de sódio 3,0 $\times$ $10^{-3} \mathrm{~mol} \mathrm{~L}^{-1}$, em $\mathrm{H}_{2} \mathrm{SO}_{4} 0,5 \mathrm{~mol} \mathrm{~L}^{-1}, f=10 \mathrm{~Hz}, \mathrm{a}=20 \mathrm{mV}, \Delta \mathrm{E}_{\mathrm{s}}=2 \mathrm{mV}$; (a) sentido direto; (b) sentido reverso e (c) resultante 
Assim como para os clorofenóis, ${ }^{19}$ o pré-tratamento catódico do eletrodo de DDB foi de grande importância, facilitando a oxidação do ciclamato de sódio em meio ácido. A Figura 2 mostra os voltamogramas de onda quadrada (correntes resultantes) obtidos para o ciclamato de sódio sobre o eletrodo de DDB sem o pré-tratamento catódico e após o pré-tratamento catódico. Observa-se que o voltamograma obtido para o ciclamato com o eletrodo pré-tratado catodicamente apresentou uma melhor definição de pico e maior magnitude de corrente. Isso indica que após o pré-tratamento catódico o eletrodo apresenta uma maior atividade eletroquímica para a ocorrência da reação de oxidação do ciclamato. Aumento similar da atividade eletroquímica do sistema, após um pré-tratamento catódico potenciostático da superfície do DDB, foi relatado anteriormente por Suffredini et al. ${ }^{19}$ para outras espécies redox.

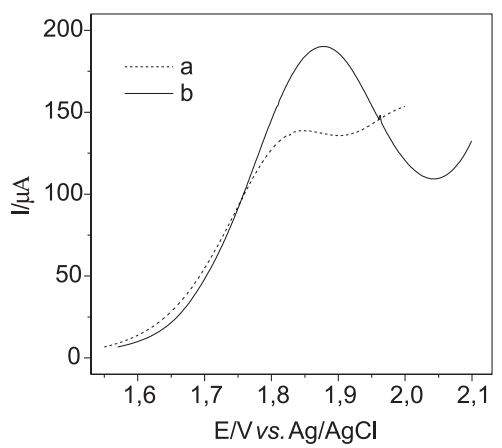

Figura 2. Voltamogramas de onda quadrada para ciclamato de sódio 3,0 $010^{-3}$ mol $L^{-1}$ empregando-se o eletrodo DDB sem pré-tratamento catódico (a) e com o pré-tratamento catódico (b), para $f=10 \mathrm{~Hz}, \mathrm{a}=20 \mathrm{mVe} \Delta E_{\mathrm{s}}=2 \mathrm{mV}$

\section{Otimização dos parâmetros experimentais}

No desenvolvimento de um método eletroanalítico faz-se necessário otimizar os parâmetros que possam influenciar a resposta voltamétrica. Um estudo preliminar empregando as técnicas voltamétricas de pulso diferencial e de onda quadrada para a determinação de ciclamato foi realizado. A sensibilidade obtida com as duas técnicas foi praticamente a mesma; entretanto, a voltametria de onda quadrada apresentou picos de menor largura e curva analítica com maior linearidade. Assim, optou-se por usar a voltametria de onda quadrada, para a qual foi realizado, então, um estudo da influência do eletrólito suporte e dos parâmetros envolvidos nessa técnica.

Experimentos iniciais com o ciclamato de sódio foram realizados em diferentes eletrólitos suportes: tampão fosfato $(\mathrm{pH}=6)$, tampão de Britton-Robinson (BR) ( $\mathrm{pH}=5$ ) e $\mathrm{H}_{2} \mathrm{SO}_{4}$ nas concentrações de 0,01 , $0,1,0,3,0,5,0,7$ e $1,0 \mathrm{~mol} \mathrm{~L}^{-1}$. O eletrólito suporte que proporcionou uma melhor resposta para o processo de oxidação do ciclamato de sódio foi a solução de $\mathrm{H}_{2} \mathrm{SO}_{4} 0,5 \mathrm{~mol} \mathrm{~L}^{-1}$ com um perfil voltamétrico mais bem definido e intensidade de pico ligeiramente maior (dados não mostrados).

A freqüência de aplicação dos pulsos de potencial $(f)$ é uma das variáveis mais importantes na voltametria de onda quadrada, pois é ela que determina a intensidade dos sinais e, conseqüentemente, a sensibilidade do método, além de fornecer informações a respeito do mecanismo eletródico envolvido no sistema. Neste estudo observou-se que à medida que se aumenta a freqüência de aplicação de pulsos de potenciais ocorre também um aumento na intensidade da corrente de pico e um deslocamento dos potenciais de pico para valores mais positivos. De acordo com a teoria desenvolvida para a voltametria de onda quadrada, ${ }^{27}$ este comportamento é característico de reações totalmente irreversíveis controladas por adsorção das espécies sobre a superfície do eletrodo. Porém, com o aumento da frequiência, há também um deslocamento da linha base para valores maiores de corrente. Portanto, a freqüência selecionada foi a de $10 \mathrm{~Hz}$, para evitar altos valores de corrente para a linha base. Um outro parâmetro estudado foi a influência da variação da amplitude de pulso de potencial $(a)$ na corrente de pico para a oxidação do ciclamato de sódio. Os resultados obtidos mostraram que, para valores de amplitudes menores que $40 \mathrm{mV}$, o aumento da intensidade de corrente é linear, sendo que amplitudes maiores parecem não atuar de modo significativo na sensibilidade para propósitos analíticos, além de haver um deslocamento nos potenciais de pico para valores menos positivos. Sendo assim, a amplitude selecionada para o desenvolvimento deste trabalho foi a de $40 \mathrm{mV}$. Na voltametria de onda quadrada a velocidade efetiva é o resultado do produto da frequiência de aplicação dos pulsos de potencial $(f)$ pelo incremento de varredura de potencial $\left(\Delta E_{\mathrm{s}}\right)$. Deste modo, um incremento de varredura maior pode aumentar o sinal obtido e melhorar a sensibilidade do método. No entanto, com incrementos maiores podem ocorrer alargamentos nos picos obtidos e, conseqüentemente, a resolução pode ser comprometida; ${ }^{28,29}$ assim, este é um parâmetro que também deve ser analisado. Neste estudo a corrente de pico variou linearmente com o incremento de varredura até $2 \mathrm{mV}$; portanto, optou-se em trabalhar com este valor de incremento de varredura.

\section{Curva analítica para o ciclamato de sódio}

Após a otimização dos parâmetros envolvidos na voltametria de onda quadrada, a curva analítica foi construída pela adição de alíquotas de $40 \mu \mathrm{L}$ da solução padrão de ciclamato de sódio $0,01 \mathrm{~mol}$ $\mathrm{L}^{-1}$ à célula eletroquímica inicialmente contendo $10 \mathrm{~mL} \mathrm{de} \mathrm{H}_{2} \mathrm{SO}_{4}$ $0,5 \mathrm{~mol} \mathrm{~L}^{-1}$. A Figura 3 apresenta os voltamogramas obtidos para as diferentes concentrações de ciclamato de sódio que variaram de 5,0 $\times 10^{-5} \mathrm{a} 4,1 \times 10^{-4} \mathrm{~mol} \mathrm{~L}^{-1}$. Observa-se que a corrente de pico aumenta proporcionalmente com o aumento da concentração e que praticamente não ocorre deslocamento no valor dos potenciais de pico. A inserção na Figura 3 corresponde à curva analítica para o ciclamato de sódio, dada pela seguinte equação de reta:

\section{$I_{\mathrm{pa}} / \mu \mathrm{A}=0,999+6,69 \times 10^{4}\left\{[\right.$ ciclamato de sódio $\left.] / \mathrm{mol} \mathrm{L}^{-1}\right\}$}

com um coeficiente de correlação de 0,9992 . O limite de detecção (três vezes o desvio padrão do branco/coeficiente angular da curva analítica) obtido utilizando o eletrodo de DDB pré-tratado catodicamente foi de $4,8 \times 10^{-6} \mathrm{~mol} \mathrm{~L}^{-1}$. No estudo da repetibilidade do eletrodo de DDB para o ciclamato de sódio, foram feitas 5 medidas sucessivas de uma solução de ciclamato de sódio $3,0 \times 10^{-3} \mathrm{~mol} \mathrm{~L}^{-1}$, sendo que

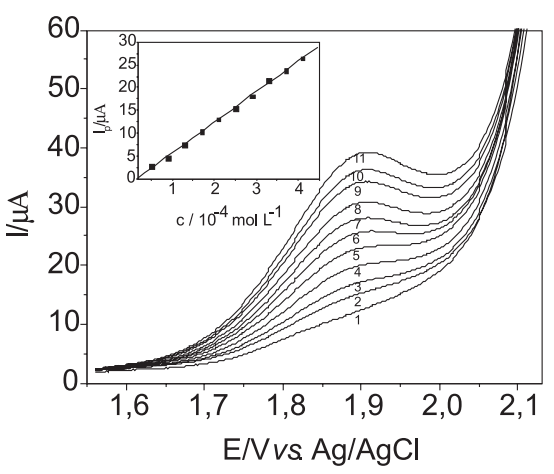

Figura 3. Voltamogramas de onda quadrada para ciclamato de sódio em diferentes concentrações: $0(1) ; 5,0 \times 10^{-5}(2) ; 9,0 \times 10^{-4}(3) ; 1,3 \times 10^{-4}(4)$; $1,7 \times 10^{-4}(5) ; 2,1 \times 10^{-4}(6) ; 2,5 \times 10^{-4}(7) ; 2,9 \times 10^{-4}(8) ; 3,3 \times 10^{-4}(9) ; 3,7$ $\times 10^{-4}(10)$ e $4,1 \times 10^{-4} \mathrm{~mol} \mathrm{~L}^{-1}(11) \mathrm{em} \mathrm{H}_{2} \mathrm{SO}_{4} 0,5 \mathrm{~mol} \mathrm{~L}^{-1}$, para $\mathrm{f}=10 \mathrm{~Hz}, \mathrm{a}$ $=20 \mathrm{mV}$ e $\Delta E_{\mathrm{s}}=2 \mathrm{mV}$ 
o desvio padrão relativo (RSD) obtido foi de $1,2 \%$. No estudo da reprodutibilidade, foram feitas medidas, em 5 dias diferentes, em soluções diferentes de ciclamato de sódio $3,0 \times 10^{-3} \mathrm{~mol} \mathrm{~L}^{-1}$, sendo que o desvio padrão relativo obtido foi de $2,4 \%$.

\section{Efeito de interferentes em potencial e teste de adição e recuperação}

A fim de investigar a seletividade do método proposto, diversas substâncias comumente adicionadas em produtos dietéticos foram analisadas: acesulfame-K, aspartame, sacarina, ácido cítrico, ácido ascórbico, sacarose, lactose, benzoato de sódio e os corantes amarelo crepúsculo, tartrazina e azul brilhante. Estes testes foram feitos pela adição destes possíveis interferentes à solução de ciclamato de sódio $3,0 \times 10^{-4} \mathrm{~mol} \mathrm{~L}^{-1} \mathrm{e}$ os resultados foram comparados com os resultados obtidos para as medidas feitas somente com a solução de ciclamato de sódio. Entre as substâncias que apresentaram eletroatividade frente ao eletrodo de DDB, apenas o acesulfame-K apresentou potencial de pico $(2,0 \mathrm{~V})$ próximo ao potencial de pico do ciclamato de sódio $(1,9$ V). Porém, a combinação destes edulcorantes não é muito empregada pelas indústrias alimentícias, e quando estes edulcorantes estão combinados em um produto dietético a concentração de acesulfame-K é bem menor que aquela de ciclamato de sódio. Estudos realizados para concentrações equimolares destes edulcorantes ([ciclamato de sódio]/[acesulfame-K] = 1) resultaram em uma interferência de $4,6 \%$. Sendo assim, o ciclamato de sódio pode ser quantificado em produtos dietéticos mesmo na presença do acesulfame-K desde que este esteja em concentrações iguais ou menores que a do ciclamato de sódio, o que normalmente é encontrado nas composições dos produtos dietéticos.

Um estudo de adição e recuperação das substâncias em amostras de interesse também foi realizado. Estes experimentos foram feitos em triplicata, por meio da adição de alíquotas de solução padrão de ciclamato de sódio às soluções das amostras dos produtos dietéticos: adoçantes de mesa (Zero $\mathrm{Cal}^{\circledR}$ e Finn ${ }^{\circledR}$ ); preparado sólido para refresco $\left(\right.$ Magro $\left.^{\circledR}\right)$ e refrigerantes de guaraná (Antarctica ${ }^{\circledR}$ e Kuat $^{\circledR}$ ).

A Tabela 1 apresenta os resultados de recuperação obtidos. Observa-se que as recuperações variaram de 98,6 a 106,0\%, indicando que a matriz de cada amostra não interfere na determinação de ciclamato de sódio quando se usa o procedimento analítico desenvolvido.

Tabela 1. Estudo de adição e recuperação de ciclamato de sódio em amostras de produtos dietéticos

\begin{tabular}{lccc}
\hline Amostras & \multicolumn{2}{c}{$\begin{array}{c}\text { Ciclamato de sódio/ } 10^{-5} \mathrm{~mol} \mathrm{~L}^{-1} \\
\text { Adicionado }\end{array}$} & $\begin{array}{c}\text { Recuperação/\% } \\
\text { Encontrado }\end{array}$ \\
\hline Zero cal $^{\circledR}$ & 1,50 & 1,48 & 98,6 \\
& 2,00 & 1,98 & 99,0 \\
Finn $^{\circledR}$ & 2,50 & 2,54 & 101,6 \\
& 1,50 & 1,57 & 104,7 \\
& 2,00 & 2,10 & 105,0 \\
Magro $^{\circledR}$ & 2,50 & 2,60 & 104,0 \\
& 1,50 & 1,48 & 98,7 \\
& 2,00 & 2,12 & 106,0 \\
Guaraná $^{\circledR}$ & 2,50 & 2,57 & 102,8 \\
Antarctica $^{\circledR}$ & 1,50 & 1,55 & 103,3 \\
& 2,00 & 2,11 & 105,0 \\
Guaraná Kuat $^{\circledR}$ & 2,50 & 2,52 & 100,8 \\
& 1,50 & 1,54 & 102,0 \\
& 2,00 & 2,08 & 104,0 \\
& 2,50 & 2,63 & 105,2 \\
\hline
\end{tabular}

$(\mathrm{n}=3)$

\section{Aplicação analítica}

Para avaliar o desempenho do procedimento analítico desenvolvido, determinou-se o ciclamato de sódio em diversos produtos dietéticos. O método utilizado para a determinação de ciclamato de sódio foi o de adições múltiplas de padrão. Os voltamogramas obtidos estão apresentados na Figura 4. A inserção nesta figura corresponde à curva analítica obtida.

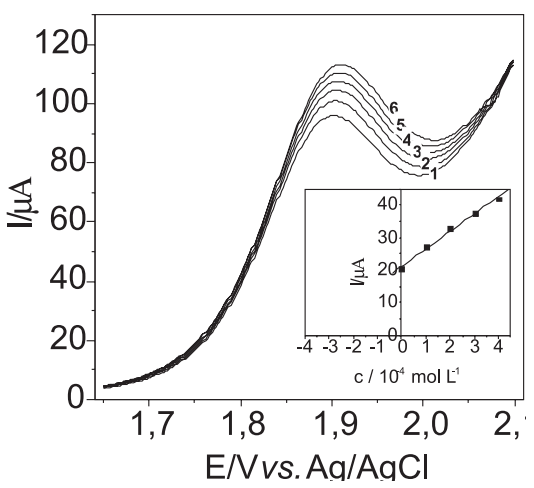

Figura 4. Voltamogramas de onda quadrada obtidos para a determinação de ciclamato de sódio em produtos dietéticos (refrigerante de guaraná Antarctica $\left.^{\circledR}\right)$. (1) amostra Antarctica ${ }^{\circledR}$; (2) amostra Antarctica ${ }^{\circledR}+5,0 \times 10^{-5}$ mol $L^{-1}$; (3) amostra Antarctica ${ }^{\circledR}+1,0 \times 10^{-4} \mathrm{~mol} \mathrm{~L}^{-1}$; (4) amostra Antarctica ${ }^{\circledR}$ $+1,5 \times 10^{-4} \mathrm{~mol} \mathrm{~L}^{-1} ;(5)$ amostra Antarctica ${ }^{\circledR}+2,0 \times 10^{-4} \mathrm{~mol} \mathrm{~L}^{-1} ;(6)$ amostra Antarctica ${ }^{\circledast}+2,5 \times 10^{-4} \mathrm{~mol} \mathrm{~L}^{-1}$ de solução padrão de ciclamato de sódio para $f=10 \mathrm{~Hz}, a=20 \mathrm{mVe} \Delta E_{s}=2 \mathrm{mV}$

A Tabela 2 apresenta os resultados obtidos para a determinação do ciclamato de sódio em produtos dietéticos empregando-se CLAE ${ }^{9,30}$ e o método voltamétrico de onda quadrada. Como pode ser observado, os resultados obtidos por ambos os métodos estão em boa concordância em um nível de confiança de $95 \%$ ( $\mathrm{t}_{\exp }=1,93$; $\left.\mathrm{t}_{\text {crítico }}=2,78, \alpha=0.05\right) .{ }^{31}$

Tabela 2. Resultados obtidos na determinação de ciclamato de sódio em produtos dietéticos analisados por CLAE e por SWV

\begin{tabular}{lccccc}
\hline Amostras & \multicolumn{2}{c}{ Ciclamato de sódio/(mg/100 mL) } \\
& $\begin{array}{c}\text { Valor } \\
\text { Rotulado }\end{array}$ & CLAE & SWV & $\begin{array}{c}\text { Erro } \\
\text { Relativo Relativo } \\
(1) / \%\end{array}$ & $\begin{array}{c}\text { Erro } \\
(2) / \%\end{array}$ \\
\hline Zero cal $^{\circledR}$ & & & & & 6,6 \\
Finn $^{\circledR}$ & 8.300 & $8.410 \pm 22$ & $8.370 \pm 23$ & 0,8 & $-0,5$ \\
Magro $^{\circledR}$ & 120 & $122 \pm 2$ & $122 \pm 4$ & 1,7 & 0 \\
Antarctica $^{\circledR}$ & 70 & $68 \pm 1$ & $72 \pm 1$ & 2,8 & 5,8 \\
Kuat $^{\circledR}$ & 31,0 & $31,5 \pm 0,5$ & $29,3 \pm 0,4$ & $-5,5$ & $-6,9$ \\
\hline
\end{tabular}

$\overline{(n=3) \text {; erro relativo }(1)=100 \times(\text { valor voltamétrico }- \text { valor rotulado }) /}$ valor rotulado; erro relativo $(2)=100 \times($ valor voltamétrico - valor CLAE)/valor CLAE

\section{CONCLUSÕES}

Os dados apresentados evidenciaram a viabilidade do emprego de um eletrodo de diamante dopado com boro pré-tratado catodicamente para a determinação de ciclamato de sódio em diferentes amostras utilizando a voltametria de onda quadrada. O ciclamato de sódio apresentou um único pico no sentido anódico em 1,9 V, com características de um processo irreversível. A curva analítica foi obtida num intervalo de concentração entre $5,0 \times 10^{-5} \mathrm{~mol} \mathrm{~L}^{-1} \mathrm{e} 4,1 \times 10^{-4} \mathrm{~mol} \mathrm{~L}^{-1}$, com um limite de detecção de $4,8 \times 10^{-6} \mathrm{~mol} \mathrm{~L}^{-1}$. A linearidade obtida 
no método proposto neste trabalho é maior que aquela obtida no método biamperométrico ${ }^{14}$ e de mesma ordem que a obtida no método amperométrico empregando um sensor com membrana lipídica. ${ }^{15}$ No entanto, neste trabalho o limite de detecção obtido $\left(4,8 \times 10^{-6} \mathrm{~mol} \mathrm{~L}^{-1}\right)$ é bem menor que os obtidos nos métodos biamperométrico ${ }^{14}(2,5 \times$ $\left.10^{-3} \mathrm{~mol} \mathrm{~L}^{-1}\right)$ e amperométrico ${ }^{15}\left(10^{-5} \mathrm{~mol} \mathrm{~L}^{-1}\right)$. Ademais, a sensibilidade obtida no método proposto foi de $6,69 \times 10^{4} \mu \mathrm{A} \mathrm{mol}^{-1} \mathrm{~L}$, enquanto que para o método biamperométrico ${ }^{14}$ foi de $2,71 \times 10^{2} \mu \mathrm{A} \mathrm{mol}^{-1} \mathrm{~L}$ e para o amperométrico, $1,59 \times 10^{3} \mu \mathrm{A} \mathrm{mol}^{-1} \mathrm{~L}^{-1}$. Por outro lado, não foi possível comparar as figuras de mérito obtidas no presente trabalho com aquelas obtidas no procedimento volumétrico proposto na literatura, ${ }^{13}$ uma vez que os autores apresentam apenas os erros relativos entre os volumes do titulante gasto (solução de nitrito de sódio) e o teor de ciclamato de soluções de referência deste edulcorante.

No estudo de adição e recuperação, foram obtidas recuperações entre 96,8 e 106,0\%, indicando que não ocorreu interferência das matrizes das amostras na determinação do ciclamato de sódio. No estudo de repetibilidade, o desvio padrão relativo obtido foi de $1,2 \%$; já no estudo de reprodutibilidade, foi de $2,4 \%$. Os resultados obtidos para a determinação de ciclamato de sódio em amostras de produtos dietéticos utilizando o procedimento proposto (SWV) estão em concordância com os resultados obtidos pelo método de referência (CLAE), em nível de confiança de $95 \%$. O procedimento desenvolvido para a determinação de ciclamato de sódio em produtos dietéticos também apresentou boa sensibilidade e não foi necessário qualquer pré-tratamento das amostras analisadas. Finalmente, cabe ressaltar que o método proposto apresenta algumas vantagens sobre o método de referência (CLAE), como, por exemplo, baixo custo, menor tempo de análise e dispensa de qualquer tratamento prévio das amostras.

\section{AGRADECIMENTOS}

Os autores agradecem o apoio financeiro e as bolsas da CAPES, FAPESP e CNPq.

\section{REFERÊNCIAS}

1. Fatibello-Filho, O.; Vieira, I. C.; Gouveia, S. T.; Calafatti, S. A.; Santos, G. A. J. M.; Quim. Nova 1996, 19, 248.

2. Pinheiro, M. V.; Oliveira, M. N.; Penna, A. L. B.; Tamine, A. Y.; International Journal of Dairy Technology 2005, 4, 193.

3. Ahmed, F. E.; Thomas, D. B.; Crit. Rev. Toxicology 1992, 22, 81.

4. O'Brien, L.; Gelardi, R. C.; Chemtech 1981, maio, 274.

5. Lawrence, J.; Analyst 1987, 112, 879.
6. Huang, Z.; Ma, J.; Chen, B.; Zhang, Y.; Yao, S.; Anal. Chim. Acta 2006, 555, 233.

7. Choi, M. M. F.; Hsu, M. Y.; Wong, S. L.; Analyst 2000, 125, 217.

8. Prando, J.; Prado, M. A.; Resumos do XIV Congresso Interno de Iniciação Científica, Campinas, Brasil, 2006.

9. Casals, I.; Reixach, M.; Amat, J.; Fuentes, M.; Serra-Majem, L.; J. Chromatogr., A 1996, 750, 397.

10. Hoo, D.; Hu, C.; Anal. Chem. 1972, 44, 2111.

11. Rocha, F. R. P.; Rodenas-Torralba, E.; Morales-Rubio, A.; Guardia, M.; Anal. Chim. Acta 2005, 547, 204.

12. Cabero, C.; Saurina, J.; Hernandez-Cassou, S.; Anal. Chim. Acta 1999, 381, 307.

13. Richardson, M. L.; Luton, P. E.; Analyst 1966, 91, 522.

14. Fatibello-Filho, O.; Capelato, M. D.; Calafatti, S. A.; Analyst 1995, 120, 2407.

15. Nikolelis, D. P.; Pantoulias, S.; Electroanalysis 2000, 12, 786.

16. Pedrosa, V. A.; Codognoto, L.; Avaca, L. A.; Quim. Nova 2003, 26, 844.

17. Barros, R. C. M.; Ribeiro, M. C.; An-Sumodjo, P. T.; Julião, M. S. S.; Serrano, S. H. P.; Ferreira, N. G.; Quim. Nova 2005, 28, 317.

18. Pleskov, Y.V.; J. Anal. Chem. 2000, 55, 1045.

19. Suffredini, H. B.; Pedrosa, V. A.; Codognoto, L.; Machado, S. A. S.; Rocha-Filho, R. C.; Avaca, L. A.; Electrochim. Acta 2004, 49, 4021.

20. Swain, G. M. Em Electroanalytical Chemistry; Bard, A. J.; Rubinstein, I., eds.; Marcel Dekker: Nova Iorque, 2004, vol. 22.

21. Hupert, M.; Muck, A.; Wang, J.; Stotter, J.; Cvakova, Z.; Haymond, S.; Show, Y.; Swain, G. M.; Diamond Relat. Mater. 2003, 12, 1940.

22. Avaca, L. A.; Codognoto, L.; Machado, S. A. S.; Diamond Relat. Mater. 2002, 11, 1670 .

23. Zhang, Y.; Yoshihara, S.; J. Electroanal. Chem. 2004, 573, 327.

24. Pleskov, Y. V.; Russ. J. Electrochem. 2002, 38, 1275.

25. Salazar-Banda, G. R.; Andrade, L. S.; Nascente, P. A. P.; Pizani, P. S.; Rocha-Filho, R. C.; Avaca, L. A.; Electrochim. Acta 2006, 51, 4612.

26. Carvalho, A. E. de; Tese de Doutorado, Universidade Federal de São Carlos, Brasil, 2007.

27. Lovric, M.; Komorsky-Lovric, S.; Murray, R. W.; Electrochim. Acta 1988, 33, 739 .

28. Souza, D.; Machado, S. A. S.; Avaca, L. A.; Quim. Nova 2003, 26, 81.

29. Codognoto, L.; Machado, S. A. S.; Avaca, L. A.; Diamond Relat. Mater. 2002, 11, 1670 .

30. Demiralay Çubuk, E.; Ozkan, G.; Guzel-Seydim, Z.; Chromatographia 2006, 63, 91 .

31. Anderson, R. L.; Practical Statistics for Analytical Chemists, Van Nostrand Reinhold: Nova Iorque, 1987. 